\title{
An Electronic Health Record Approach to Understanding Drug to Drug Interactions and Associated Knowledge Gaps in Intergrated Care of Multimorbidity
}

\author{
George DESPOTOU ${ }^{\mathrm{a}, 1}$ and Theodoros N. ARVANITIS ${ }^{\mathrm{a}}$ \\ anstitute of Digital Healthcare, WMG, University of Warwick, UK
}

\begin{abstract}
Multimorbid patients are prescribed a number of medications in parallel, which may often interact with each other, resulting in adverse effects. However, clinical guidelines on prescription of medications predominantly focus on individual conditions do not consider the guidance in the context of other guidelines, resulting in conflicts. C3-Cloud is an integrated care architecture managing multimorbidity, which amongst others, provides clinical decision support, based on reconciled guidelines, and active monitoring of drug interactions. To identify the severe interactions that resulted from multimorbidity management, in order to reevaluate guidelines as well as to identify knowledge gaps in prescribing practice. Method: Descriptive statistical analysis of interactions identified by the C3-Cloud clinical decision support, collected from the C3-Cloud FHIR repository. As part of a feasibility study, a number of interactions were identified, along with variable practice in how chemicals are represented in the EHR. 191 known severe interactions were identified. The Atorvastatin/Verapamil interaction was the most frequent. The approach has identified a number of interactions where the severity was not available, highlighting the need for further clinical review.
\end{abstract}

Keywords. Patient safety, drug to drug interactions, polypharmacy, integrated care, multimorbidity, electronic health records

\section{Introduction}

Multi-morbidity creates diverse, and sometimes, contradictory needs, which challenge patients and the delivery of health services [1]. The clinical management of patients with multi-morbidity is much more complex and time-consuming than that of those with single diseases [2]. Uncoordinated multiple treatment regimens to address multimorbidity often result in unmanaged poly-pharmacy, which leads to increased potential for adverse drug interactions and poor adherence to treatment and medication [3]. This may result in Adverse Drug Events (ADE), which may be mistakenly seen as a separate diagnostic event and treated with more medications [4]. The paper is analyzing early results of a clinical decision support system, incorporating drug interaction advisories, used as part of an integrated care architecture for management of multimorbidity.

${ }^{1}$ Corresponding Author, George Despotou, Institute of Digital Healthcare, WMG, University of Warwick, CV4 7AL, Coventry UK, E-mail: g.despotou@warwick.ac.uk 
C3-Cloud is an e-health ICT system, offering integrated, patient-centered care, considering all aspects of multi-morbidity, creating a collaborative environment for all involved stakeholders [5]. C3-Cloud was deployed in 3 pilot sites in: Basque country, Spain; region Jämtland Härjedalen, Sweden; and Warwickshire, UK. The core of the system consists of the patient care plan, a digital shared picture of the patients' needs and care goals. The care plan allows all professionals to review and understand the implications of one condition in the presence of others. This by its nature is a complex task, containing a considerable amount of diverse information. Drug safety in C3-Cloud is achieved by two means: a) offline (static) reconciliation of guidelines by clinicians, and b) dynamic drug interaction advisories provided by the C3-Cloud Drug Interaction Advisory Service (DIAS) [6], as part of its clinical decision support suite. Figure 1 shows an example of how the request to the DIAS service is made. A series of Anatomical Therapeutic Chemical (ATC) [7] codes to be checked (J01CA04, B01AA03, G03XA01) are sent to the service via an http request, and a list of interactions amongst them is returned in JSON. This occurs when physicians enter a new prescription to the system. If an interaction is detected, its effect is presented to physicians, who have the option to commit to, or amend the prescription.

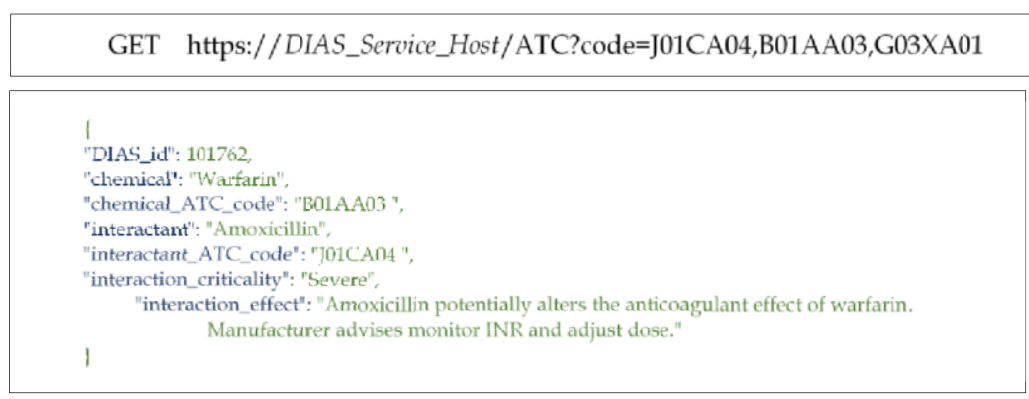

Figure 1. a) A typical DIAS GET request (top) b) extract from interactions returned in JSON (bottom).

The C3-Cloud implementation, only returns interactions classified as severe. This was considered appropriate and manageable information load presented to physicians [8].

Table 1. The guidelines used in C3-Cloud development and technology demonstrator.

\begin{tabular}{lll}
\hline \multicolumn{1}{c}{ Condition } & NICE & \multicolumn{1}{c}{ Local Guideline } \\
\hline Diabetes & NG28 & $\begin{array}{l}\text { Osakidetza, Nationella riktlinjer för diabetesvård, Swedish Medical Products } \\
\text { Agency drug treatment for type 2 Diabetes, American Diabetes Association and } \\
\text { the European Association for the Study of Diabetes (ADA/EASD) on } \\
\text { hyperglycemia, Joint British Diabetes Society (JBDS) guidelines, Scottish } \\
\text { Intercollegiate Guidelines Network (SIGN 116) guideline on diabetes } \\
\text { management. }\end{array}$ \\
$\begin{array}{lll}\text { Chronic } \\
\text { Failure }\end{array}$ & CG108 & $\begin{array}{l}\text { GuíaSalud, Nationella riktlinjer för hjärtsjukvård, European Society for } \\
\text { Cardiology, British Society for Heart Failure, 'Guidelines'. }\end{array}$ \\
$\begin{array}{l}\text { Chronic Kidney } \\
\text { Disease }\end{array}$ & CG182 & $\begin{array}{l}\text { Swedish association of nephrologists, International Kidney Disease/Improving } \\
\text { Global Outcomes - KDIGO Guidelines, Renal Association guidelines. }\end{array}$ \\
Depression & CG90 & GuíaSalud, Nationella riktlinjer för vård vid depression och ångestsyndrom. \\
\hline
\end{tabular}

Hence, the ATC codes in the responses is a smaller subset of the codes in the request. Table 1 summarizes the guidelines used in C3-Cloud. Through a co-production process, the UK National Institute of Care Excellence (NICE) guidelines were adapted to 
incorporate local practice, resulting in the $\mathrm{C} 3$-Cloud guidelines representing best common practice. The guidelines were analyzed for conflicts which were reconciled, and implemented as automated clinical decision support. Throughout the pilot, the users were informed by the clinical decision support system about the recommended way forward, based on the C3-Cloud guidelines. The final decision was made by the physician who may follow the recommendation, or use existing practice.

\section{Objectives and Method}

The objective is to analyze the interactions in order to understand: a) the most common drug risks associated with the $\mathrm{C} 3$-Cloud comorbidities, b) report to clinicians risks that were not addressed during offline reconciliation of guidelines, c) identify and report prescription habits of professionals that may not be covered by the guidelines, and d) identify knowledge gaps that will need to be bridged in order to inform practice.

The responses of the drug interaction advisory service where analyzed, in order to identify the severe (i.e. life threatening or with permanent effects) interactions that were returned to system users. A total of 150 requests were analyzed that resulted in 357 interactions. A further 166 interactions without available severity, were removed (although presented to users in C3-Cloud as they may result in severe effects), resulting in 191 known to be severe, interactions.

\section{Results}

Table 2 presents the 10 most common identified interactions, along with their potential effect and the evidence basis. All but three of the interactions are based on theoretical or anecdotal evidence.

Table 2. The 10 most common interactions, the potential effect or increased risk of effect, and evidence basis for each interaction.

\begin{tabular}{lllll}
\hline & \multicolumn{1}{c}{ Interactants } & \% & \multicolumn{1}{c}{ Potential effect } & Evidence \\
\hline 1 & Atorvastatin/Verapamil & 8.6 & Increases exposure & Study \\
2 & Bendroflumethiazide/Venlafaxine & 4.6 & Causes hypokalaemia & Theoretical \\
3 & Clopidogrel/Pioglitazone & 4.6 & Increases exposure & Study \\
4 & Bendroflumethiazide/Calcium & 2 & Risk of hypercalcaemia & Study \\
& gluconate & & & \\
5 & Bendroflumethiazide/Fluconazole & 2 & Causes hypokalaemia & Theoretical \\
6 & Bumetanide/Fluconazole & 2 & Causes hypokalaemia & Theoretical \\
7 & Bumetanide/Venlafaxine & 2 & Causes hypokalaemia & Theoretical \\
8 & Calcium & 2 & Risk of hypercalcaemia & Anecdotal \\
& gluconate/Chlorothiazide & & & \\
9 & Calcium gluconate/Chlortalidone & 2 & Risk of hypercalcaemia & Anecdotal \\
10 & Calcium gluconate/Clopamide & 2 & Risk of hypercalcaemia & Anecdotal \\
\hline
\end{tabular}

Table 3 shows the ATC codes of the drug that was being checked as part of a new prescription (based on all 357 interactions). It can be seen that the various medications were captured using various ATC levels, with the most common being drugs used in diabetes (A10 therapeutic subgroup), followed by ACE inhibitors (A09 therapeutic subgroup). Table 4 shows the ATC codes of the drugs that a potentially new prescription was checked against, showing the most common drugs already present in the patients' Electronic Health Record (based on all 357 interactions). It is worth noticing that existing 
drugs, were coded using multiple ATC groups, indicating the preference of the physicians to prescribe at the therapeutic group in some cases.

Table 3. Percentage frequency of ATC codes that were requested to be checked for associations, and the ATC hierarchy level they correspond to.

\begin{tabular}{llll}
\hline ATC Code & \multicolumn{1}{c}{ ATC Class } & \multicolumn{1}{c}{ ATC Level } \\
\hline A10BG03 & $13.3 \%$ & Pioglitazone & 5 - chemical substance \\
A10BH & $13.3 \%$ & Dipeptidyl peptidase 4 (DPP-4) inhibitors & 4 - chemical subgroup \\
C08 & $10 \%$ & Calcium channel blockers & 2 - therapeutic subgroup \\
C09 & $6.7 \%$ & ACE inhibitors & 2 - therapeutic subgroup \\
C10AA05 & $6 \%$ & Atorvastatin & 5 - chemical substance \\
A10BA02 & $4 \%$ & Metformin & 5 - chemical substance \\
C09A & $4 \%$ & ACE inhibitors & 3 - pharmacological subgroup \\
A10BB & $3.3 \%$ & Sulfonylureas & 4 - chemical subgroup \\
B01AC & $2.7 \%$ & Platelet aggregation inhibitors excl. heparin & 4 - chemical subgroup \\
C03 & $2.7 \%$ & Diuretics & 2-therapeutic subgroup \\
\hline
\end{tabular}

Table 4. Percentage frequency of ATC codes already present in the patients' record at the time of the request.

\begin{tabular}{lcll}
\hline ATC Code & $\mathbf{\%}$ & \multicolumn{1}{c}{ ATC Class } & \multicolumn{1}{c}{ ATC Level } \\
\hline A10BA02 & $7.9 \%$ & Metformin & 5 - chemical substance \\
B01AC06 & $7.3 \%$ & Acetylsalicylic acid & 5 - chemical substance \\
C10AA05 & $5.4 \%$ & Atorvastin & 5 - chemical substance \\
C08 & $5.1 \%$ & Calcium channel blockers & 2 - therapeutic subgroup \\
C03 & $4.8 \%$ & Diuretics & 2 - therapeutic subgroup \\
C09 & $4.7 \%$ & ACE inhibitors & 2 - therapeutic subgroup \\
A10A & $4.5 \%$ & Insulins and analogues & 3 - pharmacological subgroup \\
D03 & $4.3 \%$ & Dermatologicals & 2 - therapeutic subgroup \\
C07 & $3.9 \%$ & Beta blocking agents & 2 - therapeutic subgroup \\
C07AB03 & $3.8 \%$ & Atenolol & 5 - chemical substance \\
\hline
\end{tabular}

Table 5 presents the evidence basis of the 191 severe interactions. The majority of interactions relies on theoretical evidence (theoretical predictions, in vitro evidence, or by referencing other substances of the same group), whereas $21 \%$ relies on studies and a $14 \%$ on anecdotal evidence (single or limited number of event reports).

Table 5. Evidence basis of the identified interactions.

\begin{tabular}{ll}
\hline Evidence Basis & Percentage \\
\hline Anecdotal & $14 \%$ \\
Study & $21 \%$ \\
Theoretical & $65 \%$ \\
\hline
\end{tabular}

\section{Discussion and Conclusions}

The analysis based on this feasibility study identified 357 severe interactions. With the exception of the top 3 interactions, the rest were had a frequency of $2 \%$ or lower. However, even an interaction of lower frequency may affect a significant number of patients, when considering the entire population. Furthermore, it is worth considering that in the case of $\mathrm{C} 3$-Cloud, clinicians had already reconciled guidelines before the system's implementation. This seeds some major questions about the extent of the problem, as most multimorbidity patients do not manage their conditions using reconciled guidelines, but the traditional segregated approach. The findings constitute early evidence of an issue that persists. Attempting to pre-reconcile all potential 
interactions may not be practical, hence making clinical decision support in combination with access to the patient's electronic health record, a valuable prescription tool.

The approach has identified a number of interactions where the severity was not available. Although these were shown to the C3-Cloud physicians, they were not included in the results presented here, as we can't know how many of them actually are severe. Further work will need to provide evidence for these interactions. Understanding the physicians' decision making, in light of the interaction information remains to be studied. Additionally, only a $21 \%$ of interactions were based on evidence stemming from studies, with a $14 \%$ relying on anecdotal evidence. This further highlights the need to understand the confidence that we have in each type of evidence, as well as whether stronger evidence is necessary in order to inform clinical practice. Weaker evidence may be justifiably acceptable to inform guidelines [9]. The ATC codes show the trends on how new medication is represented in the system, and whether using (in some) high level ATC group (e.g., main anatomical group) is clinically equivalent to more detailed groups (e.g., chemical substance). It should be noted that the interactions represent practice from 3 different sites in 3 different countries. Overall, the approach has managed to identify a number of potential issues to be further studied as part of the clinical practice of multimorbidity management.

\section{Acknowledgements}

C3-Cloud is a project that has received funding from the European Union's Horizon 2020 research and innovation program under grant agreement No 689181. We would like to thank the C3-Cloud Clinical Reference Group and the Consortium for their contributions on the requirements specification for the C3-Cloud DIAS. The work has been partially funded by HDR UK.

\section{References}

[1] Piette, J, Richardson C and Valenstein M. Addressing the needs of patients with multiple chronic illnesses: the case of diabetes and depression, Am J Manage Care, 2004;10:152-62.

[2] Australian Institute of Health and Welfare. Comorbidity of cardiovascular disease, diabetes and chronic kidney disease in Australia, August 2007.

[3] Dumbreck S, Flynn A, Nairn M, Wilson M, Treweek S, Mercer Swart W et al. Drug-disease and drugdrug interactions: systematic examination of recommendations in 12 UK national clinical guidelines BMJ 2015;350:h949

[4] Gatenby J, Blomqvist M, et al. Adverse events targeted by drug-drug interaction alerts in hospitalized patients, International Journal of Medical Informatics 2020; 143.

[5] Erturkmen GBL, Yuksel M, Sarigul B, Arvanitis TN, Lindman P, Chen R, et al. A Collaborative Platform for Management of Chronic Diseases via Guideline-Driven Individualized Care Plans, Computational and Structural Biotechnology Journal 2019;17:869-885.

[6] Despotou G, Yuksel M, Sarigul B, Arvanitis TN. Drug Interaction Advisory Service for Clinical Decision Support of Multimordity Patient Centric Care Plans in the C3-Cloud System. Stud Health Technol Inform. 2019 Jul 4;262:388-391.

[7] ATC/DDD Index 2021, Available at: https:/www.whocc.no/atc_ddd_index/, Accessed January 1, 2021.

[8] Malhani MA et al. PMD114 - Impact of the Hitech Act's Incentive Program on the Adoption of DrugDrug Interaction Alerts and Electronic Prescribing in the Ambulatory Care Setting, Value in Health 2018;21:S178

[9] Rekić D, Reynolds KS, Zhao P, Zhang L, Yoshida K, Sachar M, Piquette Miller M, Huang SM, Zineh I. Clinical Drug-Drug Interaction Evaluations to Inform Drug Use and Enable Drug Access. J Pharm Sci. 2017 Sep;106(9):2214-2218. 